\title{
Evaluation of Acellular Dermis for Closure of Abdominal Wall Defects in a Rat Model
}

\author{
A. Pirayesh $^{a}$ A.H.M. Dur ${ }^{b}$ N.J. Paauw ${ }^{d} \quad$ S. Monstrey ${ }^{a} \quad$ R.W. Kreis ${ }^{b}$ \\ M.J. Hoekstra ${ }^{c}$ C.D. Richters ${ }^{c} d$ \\ a Plastic Surgery, UZ Gent, Gent, Belgium; ${ }^{b}$ Red Cross Hospital and ${ }^{\mathrm{C} E u r o}$ Skin Bank, Beverwijk, and ${ }^{\mathrm{d}}$ Department of \\ Molecular Cell Biology, Medical Faculty, Vrije Universiteit Medical Centre, Amsterdam, The Netherlands
}

\section{Key Words}

Dermis, human $\cdot \mathrm{NaOH} \cdot$ SureDerm ${ }^{\mathrm{TM}}$

\begin{abstract}
Background: Abdominal wall repair can be performed with synthetic or biological materials. Biological materials may reduce the risk of infections and fibrosis. The aim of this study was to evaluate two acellular human dermis products. $\mathbf{M a}$ terials and Methods: A rat model was used to compare the two materials. One was prepared using low concentrations of $\mathrm{NaOH}$; the other material was SureDerm ${ }^{\mathrm{TM}}$, which is commercially available. Full thickness defects were prepared in the abdominal wall and closed with the materials. Rats were sacrificed at 1 or 4 months after operation and the numbers of adhesions to the bowels were scored. Samples were taken for histological analysis and to measure the breaking strength. Results: In both groups a good functional integration of the implants with the abdominal wall was observed. There was no adhesion formation with the bowels in the group with the $\mathrm{NaOH}$ prototype. In the SureDerm group, 4 out of 7 rats showed only small adhesions at 4 months after operation. Breaking strength of the healed tissue was significantly higher in the $\mathrm{NaOH}$ prototype group at 4 months after operation $(p<0.0026)$. Conclusions: The results indicate that both human acellular dermis products may be used in clinical trials for closure of abdominal wall defects.
\end{abstract}

Copyright $\odot 2008$ S. Karger AG, Basel

\section{Introduction}

Abdominal wall defects resulting from trauma or incisional hernias are difficult to close for general surgeons and plastic surgeons. The prevalence is up to $10 \%$ of all laparatomies and these defects cause mechanical and physiological disturbances that increase patient morbidity. Small hernia repair by primary closure with suture has been associated with recurrence rates of up to $63 \%$ in a 7-year median follow-up. The introduction of mesh has resulted in a decrease of recurrence rates to approximately $30 \%$, with most of the recurrences occurring within the 1st year after repair [1]. Various methods and materials for treatment are described, such as synthetic materials like polytetrafluorethylene (PTFE) [2] or polypropylene mesh [3]. The disadvantage of these materials can be the formation of fibrosis and abdominal adhesions [4]. In addition, a higher risk of infection is associated with synthetic materials.

In patients with comorbidity such as morbid obesity, diabetes and or a history of infection, complications such as mesh extrusion, infection and fistula formation have been reported ranging from 23 to $78 \%$ [5]. Plastic surgeons have developed techniques to allow hernia closure by autologous tissue mobilization without the use of prosthetic materials, which are prone to infection and fistula formation. These techniques involve medial mobilization of the abdominal rectus muscles either with or without

\section{KARGER}

Fax +41613061234 E-Mail karger@karger.ch www.karger.com
C.D. Richters

Department Molecular Cell Biology, Medical Faculty

Vrije Universiteit Medical Centre (VUMC), Van der Boechorststraat 7

NL-1081 BT Amsterdam (The Netherlands)

Tel. +31 20444 8061, Fax +31 20444 8080,E-Mail cd.richters@vumc.nl 
separation of the components of the abdominal wall [6] or distant or free tissue transfer [7]. These techniques are also associated with recurrence rates of up to $30 \%$.

The use of biological materials together with these techniques may reduce recurrence and associated comorbidity. Positive results are reported using human tissue such as dura mater [8], amniotic membrane [9], dermis or xenogeneic materials (porcine submucosa, bovine pericardium, porcine dermis) [10]. Recent studies showed favorable results using human acellular dermis in animal models as well as in patients (ADM [11] and AlloDerm $[12,13])$.

The aim of our study was to evaluate the effectiveness of two other types of human acellular dermis to close abdominal wall defects. Acellular dermis was prepared from human donor skin using two different methods to remove the donor cells and antigenic structures. One prototype developed by our research group is intended to ensure the integrity of the collagen elastin matrix and is reproducible without the use of expensive techniques and/or storage methods. The other acellular human dermis type is SureDerm ${ }^{\mathrm{TM}}$, which is commercially available (Hans Biomed, Seoul, Korea). Both types were tested as implants to repair full thickness defects in the abdominal wall of rats. The incidence of adhesion formation, inflammatory response and the tensile strength was compared at 1 and at 4 months after operation.

\section{Materials and Methods}

\section{Preparation of the Implants}

Donor dermal tissue was recovered with a dermatome from a donor (deceased person who gave informed consent) after the removal of the first layer of skin with the epidermis. Thickness of this second layer of dermis was $0.8-1 \mathrm{~mm}$. Donor dermal cells were removed using two different methods. With one method, the dermis was incubated in $0.06 \mathrm{~N} \mathrm{NaOH}$ at room temperature for 6 weeks with shaking at regular intervals. The $\mathrm{NaOH}$ solution was replaced every week. After 6 weeks, the $\mathrm{NaOH}$ solution was neutralized using $\mathrm{HCl}$ and the tissue washed with $0.9 \% \mathrm{NaCl}$. This $\mathrm{NaOH}$ prototype acellular dermis was then stored in $85 \%$ glycerol until use in the rat experimental model. Processing in glycerol $85 \%$ of the material can be done without any effects on the structural integrity, and the glycerol has antibacterial and virucidal properties $[14,15]$.

The other material is produced according to a patent of Hans Biomed, the product SureDerm, a human acellular dermis for soft tissue repair. The material is prepared using enzymes and EDTA. Thereafter the material is freeze-dried and can be stored at 4$8^{\circ} \mathrm{C}$. For the experiments, the type of SureDerm for implant was used, with a thickness of $0.8-1 \mathrm{~mm}$. This was obtained from Hans Biomed.

Evaluation of Acellular Dermis
Samples of both materials were taken for histology to assess the integrity of the collagen and elastin matrix. Hematoxylin and eosin and Elastica van Gieson staining were used.

\section{Rat Model}

Twenty-eight rats were used (male Wistar, 180-200 g) obtained from Harlan-CPB (Zeist, The Netherlands). The local animal welfare committee of the VU Medical Centre approved the research protocol. The rats were kept under routine laboratory conditions with free access to food and water. They were kept in separate cages for 2 weeks after surgery.

Rats were anesthetized using a mixture of $\mathrm{N}_{2} / \mathrm{O}_{2}$ /isoflurane. A full thickness defect down to peritoneal cavity in the abdominal wall of 1 by $1.5 \mathrm{~cm}$ was created, which was repaired using the $\mathrm{NaOH}$ prototype as implant in 14 of the animals. SureDerm was used in the other group of 14 animals. Both materials were rinsed in $0.9 \% \mathrm{NaCl}$ before use and then sutured with PDS II (Ethicon, Johnson \& Johnson, Brussels, Belgium) to the defect with minimal tension. The skin was sutured with vicryl 3/0 (Ethicon, Johnson \& Johnson). Postoperative pain control was achieved with Temgesic (buprenorphine hydrochloride, $0.03 \mathrm{mg} / \mathrm{kg}$ rat) injected intramuscularly directly after the operation.

In both groups, 7 animals were sacrificed after 1 month, the other 7 animals at 4 months after operation. The abdominal cavity was opened carefully to score the presence of adhesions to the implants. Digital images were taken with a camera; these were used to estimate the size of the implants. Settings of the camera were the same for all animals. The animals were placed on a board of 15 by $25 \mathrm{~cm}$ and fixed at 4 points, always in the same way. The pictures were taken in such a way that the board was included to allow measurements. Thereafter, biopsies of the implants including abdominal wall without the skin were taken for histological analysis.

Biopsies for measuring breaking force were prepared only from the animals in the groups that were sacrificed at 4 months after operation. At this time point, the PDS sutures had been resorbed.

\section{Immuno(histo)chemistry}

Half of the samples were fixed in Kryofix and embedded in paraffin for routine hematoxylin-eosin staining. Hematoxylin and eosin were obtained from Gurr (BDH, Poole, UK). Sections were fixed in Baker's formalin before staining.

The other samples were frozen in liquid nitrogen. Cryosections were prepared to perform specific staining for rat macrophages using the ED-2 monoclonal antibody [16]. After fixation in acetone, slides were incubated with the primary antibody for $45 \mathrm{~min}$ at room temperature. Thereafter the slides were washed 3 times with PBS followed by incubation with a biotinylated secondary antibody (rabbit anti-mouse, Dako, Glostrup, Denmark) for $1 \mathrm{~h}$ at room temperature. After washing with PBS, slides were incubated with alkaline phosphatase-conjugated streptavidin (Vector, Burlingame, Calif., USA). Positive staining was then visualized by using alkaline phosphatase substrate containing naphthol AS-BI phosphate (Sigma, St. Louis, USA) and new fuch$\sin$ (Gurr) resulting in red staining. $1 \mathrm{~mm}$ levamisole (Sigma) was added to the substrate to block endogenous activity.

Two independent observers analyzed the stained sections. The numbers of positive cells (ED-2-positive macrophages) $/ \mathrm{mm}^{2}$ of tissue were counted using a grid measuring $1 \mathrm{~mm}^{2}$ (10 by 10 fields) 
Table 1. Number of rats showing adhesions ( $\mathrm{n}=7$ in each group)

\begin{tabular}{lllll}
\hline & & NaOH prototype & SureDerm & p value \\
\hline \multirow{2}{*}{1 month } & Bowel & 0 & 4 & 0.018 \\
& Skin & 3 & 0 & 0.051 \\
\hline \multirow{2}{*}{4 months } & Bowel & 0 & 3 & 0.051 \\
& Skin & 0 & 1 & 0.018 \\
\hline
\end{tabular}

in the ocular of the microscope. Five regions per section were counted, 2 were randomly selected on the border of the matrix implant, 3 in the center.

\section{Tensile Load Testing}

Tensile strength measurement was performed on a tensiometer (Instron 8872). Strips of tissue (width $1 \mathrm{~cm}$ ) comprised the implant with $2 \mathrm{~cm}$ abdominal wall tissue without the skin. They were cut at both sides using a device with fixed knives. This device is a plastic block with 3 blades placed at a distance of $1 \mathrm{~cm}$. By using this device, strips of tissue of the same size could be obtained. Stretching velocity was $2 \mathrm{~cm} / \mathrm{min}$.

\section{Statistical Analysis}

The $\chi^{2}$ test was used to analyze the adhesion formation data. The unpaired t test (two-tailed) was used to compare the results of the number of macrophages in the implants and the tensile strength.

\section{Results}

\section{Adhesion Formation}

Animals were checked daily for local or systemic complications. None of the rats suffered from infection or other complications related to the implants. They were euthanized at 1 and 4 months after the operation, and the presence of adhesions was scored. All rats had mild adhesion of the omentum to the borders of the implants. The group with the $\mathrm{NaOH}$ prototype implant had no adhesions of the intestines whereas in the group with the SureDerm implant, 4 out of 7 (after 1 month) or 3 out of 7 rats (after 4 months) showed adhesion of the small bowel to the implant (table 1). Although this is a significant $(\mathrm{p}=0.018$ and 0.051$)$ difference, these adhesions were only mild adhesions, as shown in figure 1, which were limited to one area. In addition, we observed in some rats adhesions of the implant to the skin, especially in the group with the $\mathrm{NaOH}$ prototype implant ( 3 out of 7 rats at 1 month, $p=0.051)$. None of the rats in the group with this implant showed adhesion to the skin at 4 months after operation and only 1 rat of the SureDerm group (not

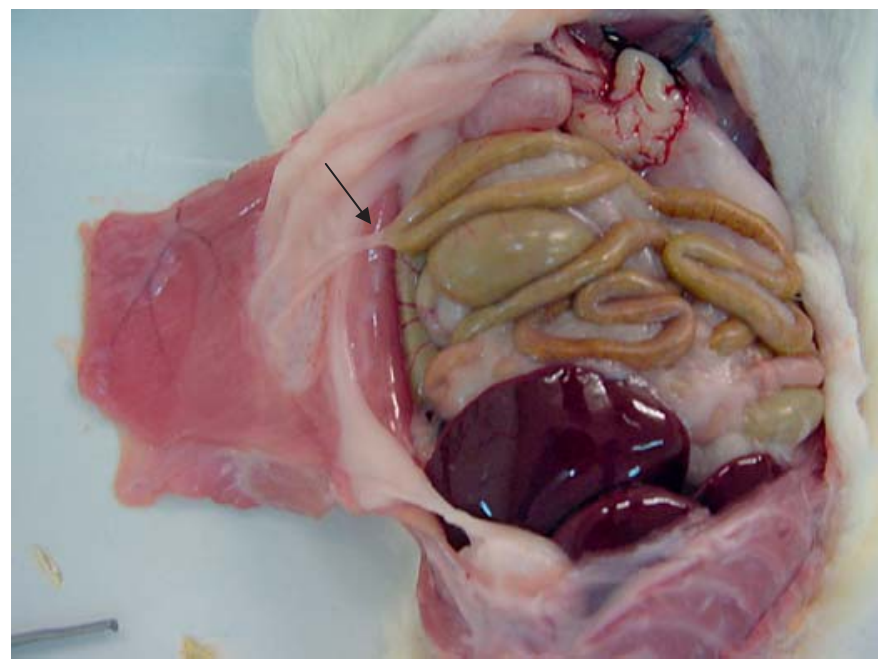

Fig. 1. Example of mild adhesion formation of the small bowel to the SureDerm implant 4 months after operation. The omentum also shows some adhesion to the implant.

significant, $p=0.299$ ). No signs of seroma adhesion could be observed in the rats with adhesions to the skin.

\section{Histopathology Results}

The sections of both materials before implantation showed intact collagen and elastin fibers. Although treatment with $\mathrm{NaOH}$ is a stringent method, the low concentration used to obtain the prototype did not result in damage to the matrix as shown earlier [17].

Good integration of the implant with the abdominal wall was observed in all rats, at 1 month after operation as well as in rats 4 months after operation. A fibrous collagen layer was present, connecting the implant with the wall (fig. 2). This layer covered the implants; some blood vessels were growing from the layer into the implant. The human-derived collagen of the implants could be easily distinguished from new rat collagen; the implant fibers are thicker and larger.

In none of the rats, relatively short fibers could be observed indicating that new collagen deposition in the implants had not taken place. The implant-derived collagen was still in situ after 4 months in the $\mathrm{NaOH}$ prototype group but there were no signs of replacement by host collagen. No clear signs of contraction of the implants could be observed. The implant was partly absorbed (as estimated from the digital pictures, up to $40 \%$ reduction of the original thickness) in 4 out of 7 rats (range $0-40 \%$ ) in the SureDerm group. 

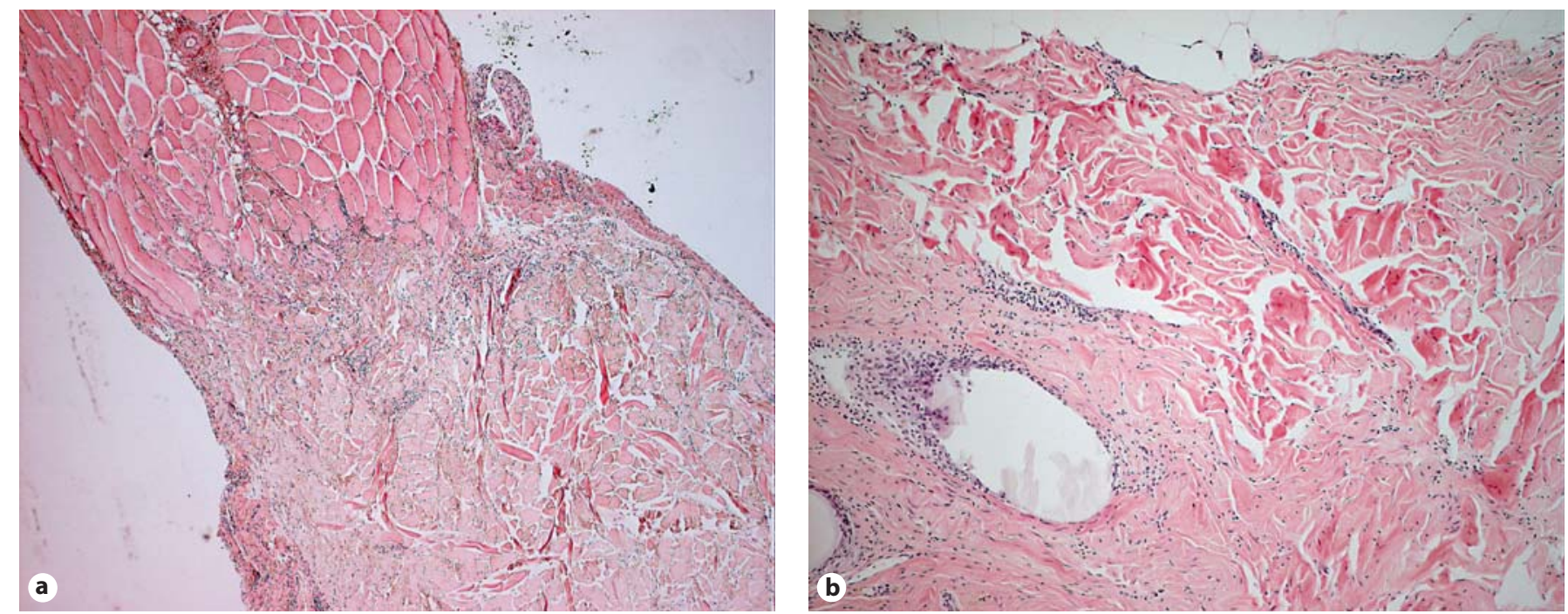

Fig. 2. HE-stained sections of the implants. A strong connection between abdominal wall and implant was formed both in the group treated with the $\mathrm{NaOH}$ prototype (a) as well as in the SureDerm group (b) 4 months after surgery.
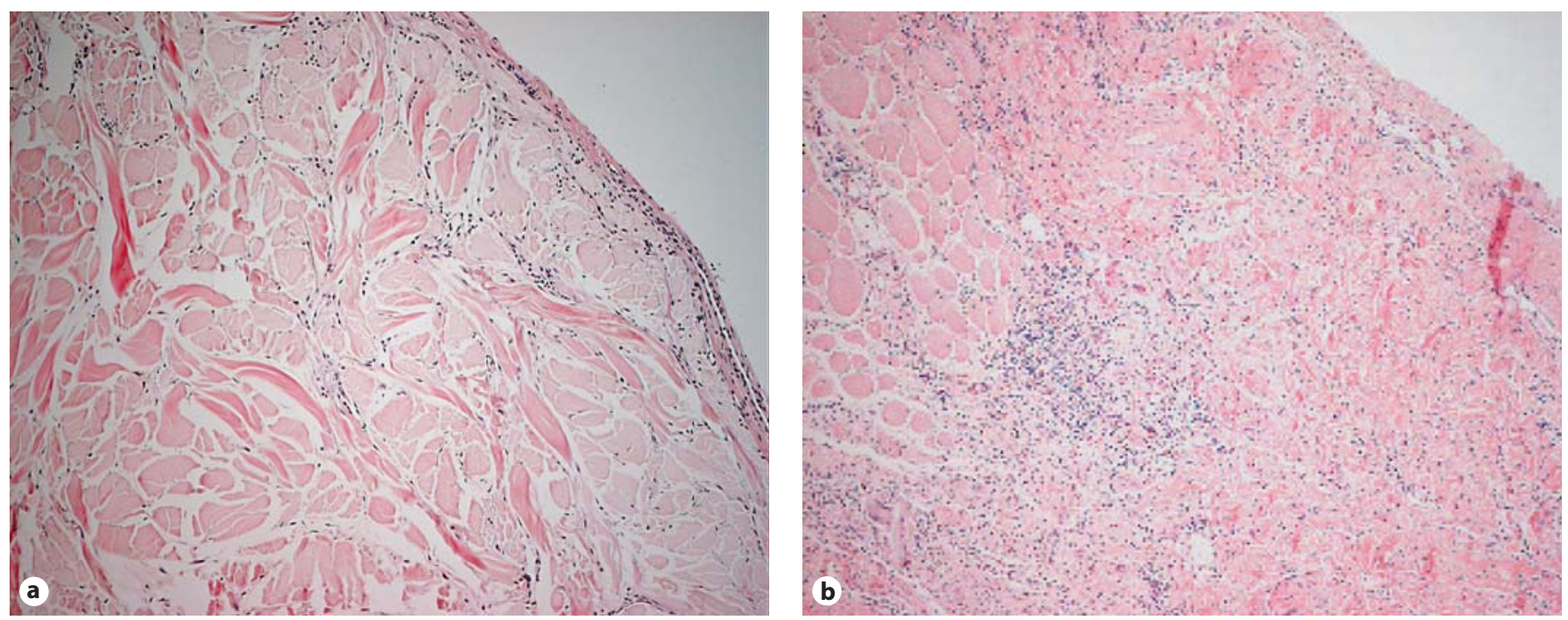

Fig. 3. HE-stained sections of the implants 4 months after surgery. More inflammatory cells are present in SureDerm (b) compared to the $\mathrm{NaOH}$ prototype (a).

Inflammatory cells (mostly mononuclear cells) were observed around sutures but also in the implant. More cells were observed in the SureDerm implants compared to the $\mathrm{NaOH}$ implants (fig. 3). Specific staining for macrophages (ED-2) showed that these cells were present in the implants (fig. 4); the number of cells was significantly higher in the SureDerm implants (fig. 5).
Blood vessels and fibroblasts were also present in the implants; higher numbers (up to 2-3 times higher) were observed in the SureDerm implants. In the $\mathrm{NaOH}$ implants, some calcification was observed around the area with sutures. 

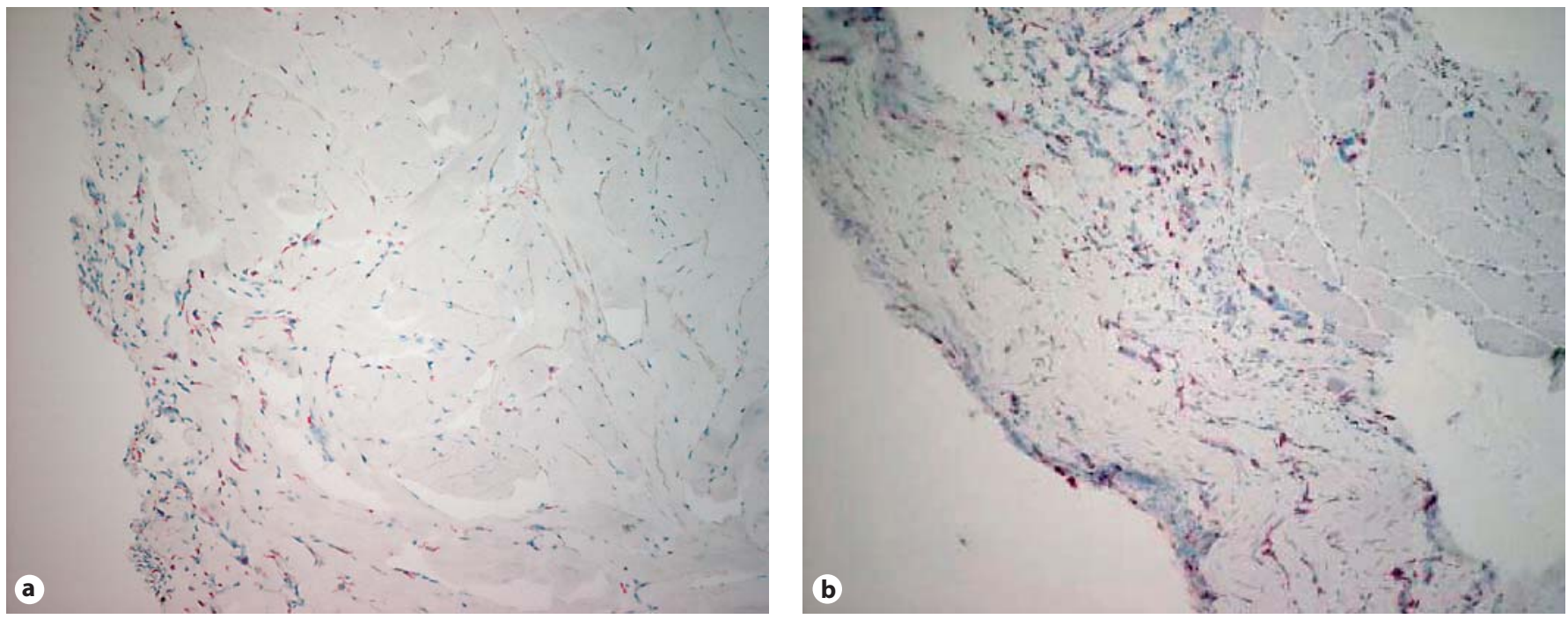

Fig. 4. Sections of the implants stained with the ED-2 antibody, recognizing rat macrophages (red cells). More cells are present in SureDerm (b) compared to the $\mathrm{NaOH}$ prototype (a) 4 months after surgery.

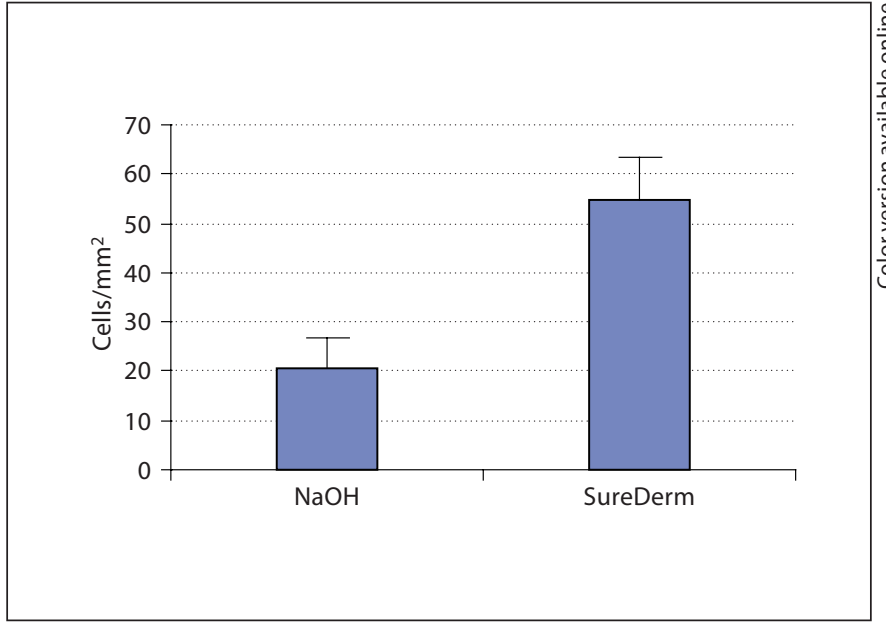

Fig. 5. The number of ED-2-positive macrophages is higher in the SureDerm implants 4 months after operation (mean $54.8 \pm 8.5$ ) compared to the $\mathrm{NaOH}$ implants (mean $20.5 \pm 6.1$ ). There is a significant difference $(\mathrm{p}<0.0001)$. Data are expressed as the mean number of cells $/ \mathrm{mm}^{2}( \pm \mathrm{SD})$ of 7 rats for each type of implant.

\section{Tensile Strength}

At 4 months after the operation, the $\mathrm{NaOH}$ implant showed a higher tensile strength compared to the SureDerm implant (fig. 6); this was a statistically significant difference ( $p<0.0026$, unpaired $t$ test). The strips of tissue tore at the border of the implants in the fibrous tissue between the implant and the tissue.

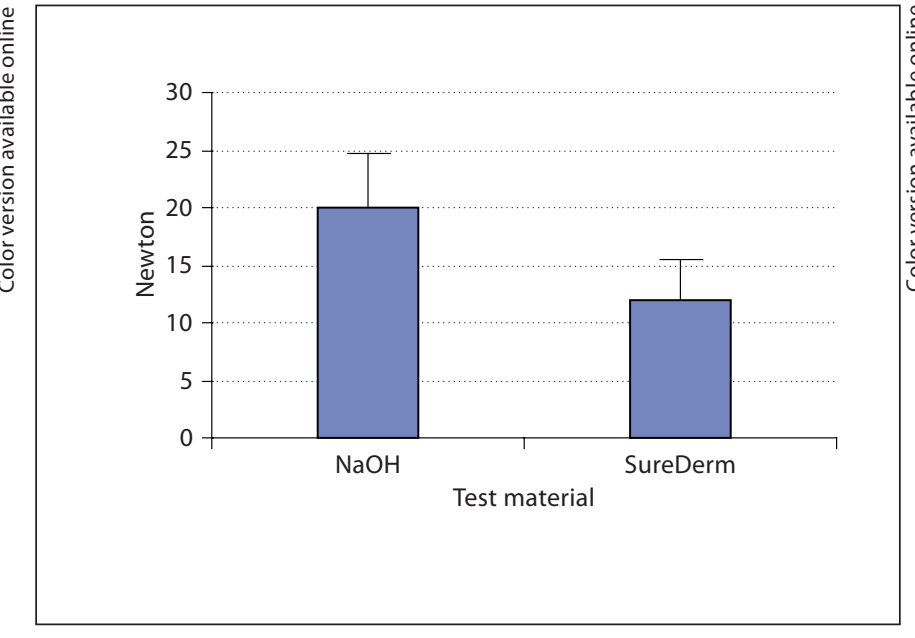

Fig. 6. The breaking strength of the healed tissue is higher in rats treated with the $\mathrm{NaOH}$ prototype implants (mean $20 \pm 4.7$ ) compared to rats with the SureDerm implants (mean $12 \pm 3.5) 4$ months after surgery. There is a significant difference $(\mathrm{p}<0.0026)$. Data shown are mean \pm SD with 7 animals in each group.

\section{Discussion}

Incisional hernia is a common problem encountered by surgeons in numerous subspecialties. Repairs that use mesh prosthesis are associated with lower recurrence rates. 
The use of mesh material with more favorable properties than traditional mesh in abdominal wall reconstruction could have a positive impact on surgical practice and patient outcomes. The ideal biomaterial would resist infection immediately after implantation, become completely remodeled into host tissue with mechanical and biological properties identical to those of the missing tissue, become rapidly revascularized and infiltrated with cells, resist seroma formation, and maintain its original strength and surface area during remodeling to prevent bulge, failure, or stretch. This ideal material has yet to be discovered or produced. Fortunately, certain bioprosthetic mesh materials have properties that may make them closer to this ideal than traditional meshes.

In the present study, we have compared two prototypes of biomaterials to close abdominal wall defects in a rat model. These biomaterials were derived from human donor skin by different methods, an $\mathrm{NaOH}$-treated prototype implant and a commercially available dermis (SureDerm). Both types of human donor skin-derived implants can be successfully used in the rat model. There was a good integration of the implant with the abdominal wall at 4 months after operation. None of the rats had complications such as infection or incisional hernia. Only mild adhesions to the omentum were formed in all rats and in some of the rats in the SureDerm group, mild adhesions to the small bowel were observed. The $\mathrm{NaOH}$ prototype induced adhesions to the skin in some of the rats at 1 month after operation but at 4 months only 1 rat of the SureDerm group showed adhesion to the skin. These are favorable results if compared to results in rats using $2 \times 3$ polypropylene mesh or PTFE with polypropylene mesh. Demir et al. [18] reported thick adhesions involving more than $50 \%$ of the material in most of the rats. We observed some adhesions of the materials to the skin without a clear explanation; this is still under investigation.

It has already been shown in animal models that $\mathrm{Al}-$ loDerm can be successfully used for abdominal wall reconstructions [13]. It can be safely used in patients for abdominal wall repair [12] but the material is expensive. In this respect, Schuster et al. [19] advised to use AlloDerm only if wound closure with skin can be achieved during operation. AlloDerm is an acellular dermal matrix derived from human donor skin [20]. It is prepared using a patented procedure by LifeCell. The results of our rat study indicate that other types of acellular dermal matrix obtained by different methods may be used with the same optimal functional results.

Evaluation of Acellular Dermis
Incubation of human skin with low concentrations of $\mathrm{NaOH}$ is a simple and cost-effective method to remove donor cells and hairs. A prototype derived from the upper dermal layer of the skin with this method $[17,21]$ can also be used to improve scar quality and to reduce contraction in burn wounds when placed underneath an autologous split skin graft and is currently undergoing phase III clinical assessment. The method to obtain SureDerm is a patented procedure of Hans Biomed and the product can be ordered for clinical use, for instance burns. So far there have been no reports of the use of this material in abdominal wall repair. Although the functional results are good for both materials in our rat model, we observed some differences. In the SureDerm implants, more of the material was resorbed at 4 months after operation; this was observed in 4 out of 7 rats. Most probably, this is due to the significantly higher number of inflammatory cells (predominantly macrophages) that were present in the SureDerm implants. The $\mathrm{NaOH}$ method may be more stringent compared to the method to obtain SureDerm, leaving fewer antigenic structures in the implants that can induce an inflammatory response. The lower breaking strength of SureDerm is most likely the result of this resorption of the material by macrophages.

We observed that on both materials, a well-organized collagen layer was formed, connecting the abdominal wall with the implant and as a parallel layer on the implant covering it. This resulted in a functional closure of the defects without complications in most of the rats indicating that the materials are suitable for use in patients. We did not observe any replacement of the collagen of the implant by new collagen produced by host fibroblast infiltrating the implant. This is in agreement with the results of Zheng et al. [10]; they described in a study on porcine dermal collagen in rats that the implant also became encapsulated rather than replaced by new tissue. This may be due to the xenogeneic origin of the implants. The human skin is made acellular and induces only a limited inflammatory response. The structures of the extracellular matrix molecules may differ from rat molecules in such way that collagen production is not induced in the rat fibroblasts. In future experiments, we will study the materials in large animals such as pigs and in an allogeneic setting, i.e. porcine skin treated with $\mathrm{NaOH}$ or with the SureDerm method before proceeding with a clinical study.

Bioprosthetic materials have increasingly become the subject of clinical and scientific interest, and their clinical use for abdominal wall reconstruction has increased over 
the last years with impressive results, particularly in adverse situations. However, a considerable amount of information has not yet been elucidated or quantified with respect to the biological and physiological mechanism of action and long-term outcomes of abdominal wall reconstruction with bioprosthetic materials. The indications and contraindications for their use have not been defined clearly and likely will become clearer with continued basic science studies and clinical outcome data. Additional evidence-based study data will allow modifications of existing materials and introduction of new products with superior properties and function, which will enhance management of abdominal wall defects.

\section{References}

1 Burger JW, Luijendijk RW, Hop WC, Halm JA, Verdaasdonk EG, Jeekel J: Long term follow-up of a randomized controlled trial of suture versus mesh repair of incisional hernia. Ann Surg 2004;240:578-583.

-2 Bauer JJ, Salky BA, Gelernt IM, Kreel I: Repair of large abdominal wall defects with expanded polytetrafluorethylene (PTFE). Ann Surg 1987;206:765-769.

- 3 Voyles CR, Richardson JD, Bland KI, Tobin GR, Flint LM, Polk HC Jr: Emergency abdominal wall reconstruction with polypropylene mesh: short-term benefits versus long-term complications. Ann Surg 1981; 194:219-223.

4 Amid PK: Intraperitoneal polypropylene mesh repair of incisional hernia is not associated with enterocutaneous fistula. Br J Surg 2000;87:1436-1437.

-5 Leber GE, Carb JL, Alexander AI, Reed WP: Long term complications associated with prosthetic repair of incisional hernias. Arch Surg 1998;133:378-382.

-6 Ramirez OM, Ruas E, Dellon AL: 'Component separation' for closure of abdominal wall defects: an anatomical and clinical study. Plast Reconstr Surg 1990;86:519-526.

7 Williams JK, Carlson GW, de Chalain T, Howell R, Coleman JJ: Role of tensor fasciae latae in abdominal wall reconstruction. Plast Reconstr Surg 1998;101:713-718.
8 Kama NA, Coskun T, Yavuz H, Dogany M, Reis E, Akat AZ: Autologous skin graft, human dura mater and polypropylene mesh for the repair of ventral abdominal hernias: an experimental study. Eur J Surg 1999;165: 1080-1085.

-9 Szabo A, Haj M, Waxsman I, Eitan A: Evaluation of Seprafilm and amniotic membrane as adhesion prophylaxis in mesh repair of abdominal wall hernia in rats. Eur Surg Res 2000;32:125-128.

10 Zheng F, Lin Y, Verbeken E, Claerhout F, Fastrez M, de Ridder D, Deprest J: Host response after reconstruction of abdominal wall defects with porcine dermal collagen in a rat model. Am J Obstet Gynecol 2004;191:19611970.

-11 An G, Walter RJ, Nagy K: Closure of abdominal wall defects using acellular dermal matrix. J Trauma 2004;56:1266-1275.

12 Buinewicz B, Rosen B: Acellular cadaveric dermis (AlloDerm): a new alternative for abdominal hernia repair. Ann Plast Surg 2004; 52:188-194.

13 Butler CE, Prieto VG: Reduction of adhesion with composite AlloDerm/polypropylene mesh implants for abdominal wall reconstruction. Plast Reconstr Surg 2004;114: 464-473.

14 van Baare J, Ligtvoet EE, Middelkoop E: Microbiological evaluation of glycerolized cadaveric donor skin. Transplantation 1998; 65:966-970.

$\checkmark 15$ Cameron PU, Pagnon JC, van Baare J, Reece JC, Vardaxis NJ, Crowe SM: Efficacy and kinetics of glycerol inactivation of HIV-1 in split skin grafts. J Med Virol 2000;60:182188.
6 Dijkstra CD, Döpp EA, Joling P, Kraal G: The heterogeneity of mononuclear phagocytes in lymphoid organs: distinct macrophage subpopulations in the rat recognized by the antibodies ED1, ED2, and ED3. Immunology 1985;54:589-599.

17 Richters CD, Pirayesh A, Hoeksema H, Kamperdijk EW, Kreis RW, Dutrieux RP, Monstrey S, Hoekstra MJ: Development of a dermal matrix from glycerol preserved allogeneic skin. Cell Tissue Bank 2008, E-pub ahead of print.

18 Demir U, Mihmanli M, Coskun H, Dilege W, Kalyoncu A, Altinli E, Gunduz B, Yilmaz B: Comparison of prosthetic materials in incisional hernia repair. Surg Today 2005;35: 223-227.

19 Schuster R, Singh J, Safadi BY, Wren SM: The use of acellular dermal matrix for contaminated abdominal wall defects: wound status predicts success. Am J Surg 2006;192:594597.

20 Livesey S, Herndorn DN, Hollyoak MA, Atkinson YH, Nag A: Transplanted acellular allograft dermal matrix. Transplantation 1995;60:1-9.

21 Richters CD, Ulrich MM, Verkerk M, Dutrieux RP, Breetveld M, Gibbs S, Hoekstra MJ, Kreis RW: Development of a dermal substitute from glycerol preserved donor skin. Wound Repair Regen 2003;11:A24. 